REGARDS

SUR L'ECONOMIE ALLEMANDE

BULLETIN ECONOMIQUE DU CRAC

\section{Regards sur l'économie allemande}

Bulletin économique du CIRAC

$91 \mid 2009$

Varia

\title{
Innovation : une priorité, surtout en temps de crise
}

\section{Solène Hazouard}

\section{OpenEdition}

\section{Journals}

Édition électronique

URL : http://journals.openedition.org/rea/3715

DOI : 10.4000/rea.3715

ISBN : 978-2-8218-0878-2

ISSN : 1965-0787

Éditeur

CIRAC

Édition imprimée

Date de publication : 1 mai 2009

Pagination : 83-84

ISSN : 1156-8992

Référence électronique

Solène Hazouard, «Innovation : une priorité, surtout en temps de crise », Regards sur l'économie allemande [En ligne], 91 | mai 2009, mis en ligne le 01 mai 2011, consulté le 15 septembre 2020. URL http://journals.openedition.org/rea/3715

Ce document a été généré automatiquement le 15 septembre 2020

(C) CIRAC 


\title{
Innovation : une priorité, surtout en temps de crise
}

\author{
Solène Hazouard
}

\section{La RFA consacre $2,54 \%$ de son PIB à la R\&D}

1 Alors que la Commission européenne a décidé de placer l'année 2009 sous le signe de l'innovation et de la créativité, l'Allemagne vient de publier deux rapports soulignant la nécessité de poursuivre les efforts entrepris en matière d'innovation, dans un pays qui a pourtant consacré $2,54 \%$ de son PIB à la R\&D en 2007, se plaçant au $4^{\mathrm{e}}$ rang mondial derrière les Etats-Unis, le Japon et la Chine (moyenne OCDE : 2,26 \%).

\section{Dépenses de R\&D du Bund : 12 milliards $€$ en 2009}

2 Le rapport « Recherche et innovation pour l'Allemagne » (« Forschung und Innovation für Deutschland. Bilanz und Perspektive »), publié fin avril 2009 par le ministère fédéral de l'Enseignement et de la Recherche (BMBF), dresse un bilan positif dela politique de la recherche et de l'innovation du gouvernement fédéral. L'augmentation conséquente, ces dernières années, des moyens alloués à la R\&D ainsi que l'introduction en 2006 de la Stratégie Hautes Technologies pour l'Allemagne (Hightech-Strategie für Deutschland) ont accru les perspectives de croissance de l'Allemagne, se félicite le BMBF. Ainsi depuis 2005, le volume des investissements annuels du Bund est passé de 9 milliards $€$ à 12 milliards $€$. Quant aux programmes conjoncturels adoptés dans le cadre de la crise actuelle, ils prévoient des investissements massifs dans l'enseignement, la recherche et l'innovation en vue de leur modernisation: 6,5 milliards $€$ pour la formation/ recherche, et 900 millions $€$ pour la R\&D dans les PME. 
Dépenses de R\&D du Bund et des Länder (financement), en millions $€$

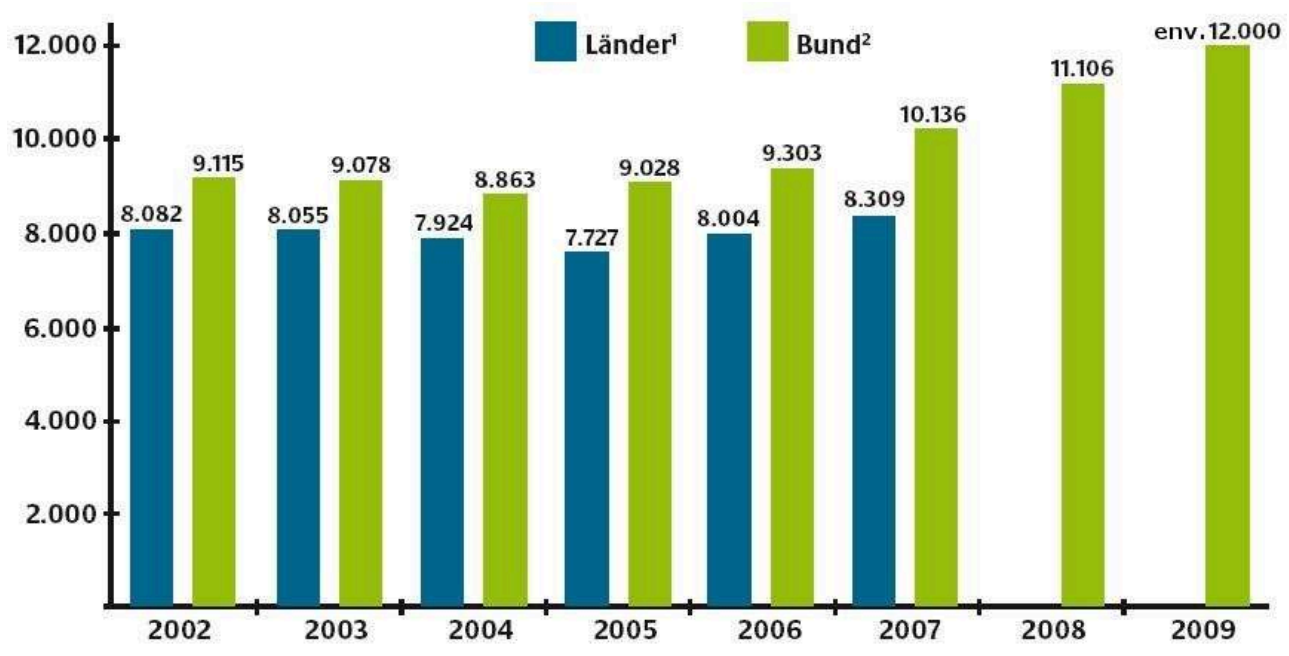

Source : BMBF. 1) 2007 : estimation ; 2) à partir de 2008 : objectif prévisionnel.

\section{Dépenses privées de R\&D : 46 milliards $€$ en 2008}

En outre, le ministère de la Recherche dresse le bilan de l'Initiative d'Excellence universitaire (Exzellenzinitiative), du Pacte de l'enseignement supérieur (Hochschulpakt) et du Pacte pour la Recherche et l'Innovation (Pakt für Forschung und Innovation; sur ces trois pactes, voir REA 88/08). Ceux-ci ont eu un impact favorable sur l'attractivité scientifique de la RFA, consolidée par ailleurs par un bon positionnement des entreprises sur les marchés mondiaux des biens technologiques. Ces dernières ont saisi elles aussi le caractère déterminant de l'innovation pour la compétitivité, accroissant leurs dépenses de R\&D de 4,4 milliards $€(+11,3 \%)$ entre 2005 et 2007 , contre à peine $3,1$ milliards $€$ entre 2000 et 2005 ( $+8,6 \%)$. Le volume total du financement privé prévu pour 2008 était de 45,7 milliards $€$, soit 2,7 milliards $€$ de plus. En raison d'une aide fédérale renforcée pour les PME, la hausse est encore plus nette au sein de cette catégorie d'entreprises, avec $+12 \%$ entre 2006 et 2007 (voir REA 90/09).

Inscrivant ses objectifs dans une vision à long terme, le gouvernement fédéral souhaite donc, malgré, ou plutôt : en raison de la crise, poursuivre dans la voie empruntée. Il importe plus que jamais de promouvoir la compétitivité des entreprises allemandes, décisive en matière d'emploi, et de contribuer à répondre aux défis actuels que sont notamment le réchauffement climatique, l'efficience énergétique, la maitrise des ressources naturelles, la santé ou le vieillissement démographique.

\section{L'effort doit être poursuivi, notamment en ce qui concerne le soutien aux PME}

En publiant ce bilan, le gouvernement fédéral prend position sur le rapport 2009 de la Commission d'experts Recherche et Innovation (Expertenkommission Forschung und Innovation, EFI), remis le 4 mars dernier à la chancelière. Ce think tank indépendant, instauré en 2006 par le gouvernement fédéral, a pour vocation de formuler des recommandations aux pouvoirs publics en matière d'innovation, cette notion allant 
bien au-delà de la R\&D pour y inclure les processus de commercialisation ou de valorisation. Bien qu'elle soutienne dans l'ensemble les mesures phares du gouvernement fédéral, la Commission d'experts estime que les mesures d'investissement dans le système d'enseignement supérieur adoptées dans le cadre du second programme conjoncturel (voir REA 90/09) doivent impérativement venir s'ajouter aux moyens prévus dans le cadre de la politique générale de modernisation du système d'enseignement et de formation. Par ailleurs, elle déplore le "faible niveau de capitaux propres détenus par les PME allemandes » et le "développement trop limité du capitalrisque » outre-Rhin, comparé à la situation internationale, qui constituent une "faiblesse essentielle du système allemand d'innovation ».

6 Fidèle à son approche globale du concept d'innovation, l'EFI préconise d'aller plus loin dans les réformes avec une refonte du système de formation, une intensification des transferts de technologies, ainsi que la mise en place d'un régime fiscal incitant à l'innovation, via notamment l'introduction d'une aide fiscale pour les activités de R\&D (crédit impôt-recherche). Outre-Rhin, la politique de l'innovation s'inscrit ainsi dans un ensemble plus vaste : celui de la politique générale de réformes structurelles destinées à renforcer la compétitivité globale du site Allemagne ("Stratégie de Lisbonne»). La crise actuelle rappelle l'urgence de cet effort de modernisation, d'autant plus, comme le souligne le rapport de l'EFI, que "la politique de recherche et d'innovation ne déploie ses effets qu'à moyen, voire à long terme »... (SH)

\section{INDEX}

Mots-clés : innovation, compétitivité, R\&D, recherche, dépenses, PME, Mittelstand, politique économique, formation 